\title{
Energy and angular momentum in strong gravitating systems
}

\author{
Roh-Suan Tung \\ Center for Astrophysics \\ Shanghai Normal University \\ Shanghai 200234, China \\ tung@shnu.edu.cn
}

\begin{abstract}
A quasilocal framework of stationary and dynamical untrapped hypersurfaces is introduced to generalize the notions of energy and angular momentum of isolated and dynamical trapping horizons to general strong gravitating systems.
\end{abstract}

\section{Introduction}

The notions of energy and angular momentum for weak gravitating systems in classical general relativity are well understood in terms of the symmetry at the asymptotically spatial infinity and the asymptotically null infinity. The question of how to define energy and angular momentum for strong gravitating systems has been raised for a while in searching for the "quasi-local energy-momentum and angular momentum" [1]. The idea is to find a suitable definition of total energy-momentum and angular-momentum, surrounded by a spacelike two dimensional surface $S$, in four dimensional spacetime $M$. The construction is quasi-local in the sense that it refers only to the geometry of $S$ (intrinsic metric, first fundamental form), the extrinsic curvatures (second fundamental forms) and the connection 1-forms on the normal bundle (normal fundamental forms) for its embedding in $M$. 
An especially interesting example of strong gravitating systems is black hole, which is now believed to be common in the universe. Traditional description of black holes in terms of event horizons is inadequate for the expected observational data. For non-stationary spacetimes, quasi-local notions of trapped and marginal surfaces have now been found to be more useful within the framework of isolated, and dynamical trapping horizons [2, 3, 4, 5, 6, 7, 8]. These frameworks enable one to significantly extend the laws of black hole mechanics to the dynamical regime with the associated notions of energy, angular momentum and their fluxes, and have been applied to several problems in mathematical general relativity, numerical relativity, and quantum gravity [9]. These progresses on black hole dynamics lead to a question whether we can generalize the conservation laws for isolated and dynamical trapping horizons to general untrapped regions so that we can study the change of energy, angular momentum and their fluxes for untrapped strong gravitating systems, e.g., before the black hole horizon was formed. The hoop conjecture for black-hole formation says that, "black holes with horizons form when and only when a mass $m$ gets compacted into a region whose circumference $C$ in every direction is $C \leq 4 \pi G m$." However, neither the mass, nor notion of the circumference is well-defined. The most natural definition should be in some sense quasilocal.

In order to understand the physical quantities in these dynamical processes, we extend the framework of isolated and dynamical trapping horizons to the untrapped regions with the notions of stationary and dynamical untrapped hypersurfaces [10]. With these notions, one can give well motivated definitions of physical quantities such as the energy and the angular momentum[10], and the fluxes [11] of energy and angular momentum of matter and gravitational radiation falling into the black holes and other strong gravitating systems.

\section{Stationary and dynamical untrapped hy- persurfaces}

We begin with the geometry of an untrapped two-surface $S$ embedded in a four-dimensional spacetime $M$. Introduce a set of orthonormal vectors $e_{0}, e_{1}, e_{2}, e_{3}$ adapted to the two-surface $S$, with $e_{0}$ and $e_{1}$ being the set of timelike and spacelike unit normals to $S$ and $e_{A}=\left(e_{2}, e_{3}\right)$ being tangent to $S$. 
The extrinsic curvatures of $S$ with respect to $e_{0}$ and $e_{1}$ directions are given by $k\left(e_{0}\right)_{A B}=g\left(e_{B}, \nabla_{A} e_{0}\right)=-\Gamma_{0 B A}$ and $k\left(e_{1}\right)_{A B}=g\left(e_{B}, \nabla_{A} e_{1}\right)=-\Gamma_{1 B A}$. The connection 1 -forms in the normal bundle are given by $\varpi_{A}=g\left(e_{1}, \nabla_{A} e_{0}\right)=$ $-\Gamma_{01 A}$. Here $\Gamma_{I J K}=-g\left(e_{J}, \nabla_{K} e_{I}\right)$ are Ricci rotation coefficients. The expansion vector $H$, and the dual expansion vector $H_{\perp}$ are defined with the trace of the extrinsic curvatures $k\left(e_{0}\right)$ and $k\left(e_{1}\right)$,

$$
\begin{aligned}
H & =k\left(e_{1}\right) e_{1}-k\left(e_{0}\right) e_{0}, \\
H_{\perp} & =k\left(e_{1}\right) e_{0}-k\left(e_{0}\right) e_{1} .
\end{aligned}
$$

These vectors are independent of choice of normal frames for the two-surface. They are uniquely defined given the two-surface $S$ and constitute a set of natural normal vectors for $S$. Unlike $H$ and $H_{\perp}$, the connection 1-forms in the normal bundle $\varpi_{A}$ depends on the choice of normal frames for the twosurface. However, for untrapped surfaces, we can use the uniquely determined unit normal vectors for the two-surface $S$,

$$
\hat{e}_{0}=\frac{H_{\perp}}{|H|}, \quad \hat{e}_{1}=\frac{H}{|H|},
$$

to fix the gauge $\left(|H|=\sqrt{k\left(e_{1}\right)^{2}-k\left(e_{0}\right)^{2}} \neq 0\right)$. So that $\varpi_{A}$ is uniquely defined [12, 13, 14].

A two-surface $S$ is trapped, untrapped, or marginal if the dual expansion vector $H_{\perp}$ is spacelike, timelike, or null respectively, everywhere on $S$. Note that, on $S$, the trace of the extrinsic curvature is zero along the direction of the dual expansion vector, i.e. $\left.k\left(H_{\perp}\right)\right|_{S}=0$, and we have

$$
\left.£_{H_{\perp}} \varrho\right|_{S}=0,
$$

where $\varrho$ is the area element of $S$. This is the key equation for the definition of the stationary untrapped hypersurfaces.

Definition 1 (stationary untrapped hypersurface) A smooth timelike hypersurface $\Delta=\mathbb{S}^{2} \times \mathbf{R}$ is said to be a dynamical untrapped hypersurface if it can be foliated by a family of closed two-surfaces $S$ such that each foliation is an untrapped surface. If on each leaf of the dynamical untrapped hypersurface, the dual expansion vector $H_{\perp}$ is tangent to the dynamical untrapped hypersurface, then it is called a stationary untrapped hypersurface $\triangle_{S}$. 
Note that it is the "hypersurface" that is "stationary". The actual spacetime can be dynamical and non-stationary. The key equation (4) for the stationary untrapped boundary conditions implies the area of a cross section of $\triangle_{S}$ is constant along $H_{\perp}$. The definition of stationary untrapped hypersurface keeps the property of 'non-expanding' and generalize the null normal used in marginal surfaces for isolated and dynamical trapping horizons, to the dual expansion vector $H_{\perp}$ for untrapped surfaces. Therefore, an alternative name for the "stationary untrapped hypersurfaces" might be "non-expanding untrapped hypersurfaces". The dual expansion vector $H_{\perp}$ plays the role for stationary untrapped hypersurfaces, which the stationary Killing vector plays for stationary black holes. In the limit when the dual expansion vector $H_{\perp}$ is null, $S$ reduces to a marginal surface, the hypersurface reduces to a nonexpanding horizon [9].

\section{Conserved quantities associated with sta- tionary untrapped hypersurfaces}

The notions of stationary untrapped hypersurfaces extract the minimal conditions which are necessary to uniquely define energy and angular momentum for untrapped strong gravitating systems. In this section, we shall derive the conserved quantities by extending the requirement of the functional differentiability of the Hamiltonian, considered first by Regge and Teitelboim [15], for spatial infinity to the finite spatial two-surfaces.

For a general diffeomorphism-invariant field theory in four dimensions with a Lagrangian 4-form $\mathcal{L}(\varphi, p)=d \varphi \wedge p-\Lambda(\varphi, p)$, where $\varphi$ denotes an arbitrary collection of dynamical fields. The equations of motion are obtained by computing the first variation of the Lagrangian.

$$
\delta \mathcal{L}=d(\delta \varphi \wedge p)+\delta \varphi \wedge \frac{\delta \mathcal{L}}{\delta \varphi}+\frac{\delta \mathcal{L}}{\delta p} \wedge \delta p
$$

For any diffeomorphism generated by a smooth vector field $\xi$, we can replace the variational derivative $\delta$ by the Lie derivative $£_{\xi}$,

$$
£_{\xi} \mathcal{L}=d\left(£_{\xi} \varphi \wedge p\right)+£_{\xi} \varphi \wedge \frac{\delta \mathcal{L}}{\delta \varphi}+\frac{\delta \mathcal{L}}{\delta p} \wedge £_{\xi} p .
$$

One can then define a conserved Noether current 3-form $J(\xi)$ by $J(\xi):=$

$£_{\xi} \varphi \wedge p-i_{\xi} \mathcal{L}$, such that the Noether current $d J(\xi) \simeq 0$ is closed on shell. 
Locally there exists a 2-form $Q(\xi)=i_{\xi} \varphi \wedge p$ (the Noether charge) such that

$$
J(\xi)=\xi^{\mu} \mathcal{H}_{\mu}+d Q(\xi) .
$$

On shell, the variation of the Noether current 3-form is given by,

$$
\delta J(\xi)=\omega\left(\varphi, \delta \varphi, £_{\xi} \varphi\right)+d i_{\xi}(\delta \varphi \wedge p),
$$

where $\omega$ is the presymplectic current 3 -form defined by $\omega\left(\varphi, \delta_{1} \varphi, \delta_{2} \varphi\right)=$ $\delta_{2} \varphi \wedge \delta_{1} p-\delta_{1} \varphi \wedge \delta_{2} p$. Its integral over a spacelike hypersurface $\Sigma$ defines the presymplectic form $\Omega$. If

$$
\Omega\left(\varphi, \delta \varphi, £_{\xi} \varphi\right) \equiv \int_{\Sigma} \omega\left(\varphi, \delta \varphi, £_{\xi} \varphi\right)=\delta \mathbb{H}(\xi)
$$

is a total variation for some function $\mathbb{H}(\xi)$ on the field space, then $£_{\xi} \mathbb{H}(\xi)=0$. $\mathbb{H}(\xi)$ is the Hamiltonian (conserved quantity) conjugate to $\xi[10,13,16,17$, [18, 19]. One can write the integrand as the exterior derivative of a 2-form. Therefore the integral is performed over the boundary $S$ of $\Sigma$,

$$
\Omega\left(\varphi, \delta \varphi, £_{\xi} \varphi\right) \equiv \oint_{S} \delta Q(\xi)-i_{\xi}(\delta \varphi \wedge p)=\delta \mathbb{H}(\xi)
$$

The conserved quantity, if it exists, is an integral over this boundary.

For general relativity, $S=\int \mathcal{L}=\int R^{a b} \wedge *\left(\vartheta_{a} \wedge \vartheta_{b}\right)$, where $R^{a b}$ is the curvature 2-form constructed by the connection 1 -form $\Gamma^{a b}$, and $\vartheta^{a}$ is the orthonormal frame 1-form field. The presymplectic form is given by

$$
\Omega\left(\varphi, \delta \varphi, £_{\xi} \varphi\right)=\oint_{S} \frac{1}{2} i_{\xi} \Gamma^{a b} \delta\left(\epsilon_{a b c d} \vartheta^{c} \wedge \vartheta^{d}\right)+\oint_{S} i_{\xi} \vartheta^{c} \wedge \delta \Gamma^{a b} \wedge \epsilon_{a b c d} \vartheta^{d} .
$$

Decompose into its normals and tangents of a two-surface boundary $S$ gives [10],

$$
\Omega\left(\varphi, \delta \varphi, £_{\xi} \varphi\right)=\oint_{S} 2 i_{\xi} \Gamma^{01} \delta \varrho-\oint_{S} 2 \varrho\left(i_{\xi} \vartheta^{0} \delta k\left(e_{1}\right)+i_{\xi} \vartheta^{1} \delta k\left(e_{0}\right)-i_{\xi} \vartheta^{A} \delta \varpi_{A}\right) .
$$

Note that the presymplectic form $\Omega\left(\varphi, \delta \varphi, £_{\xi} \varphi\right)$ depends only on the variation of the area element (together with the variation of the second and normal fundamental forms). Not all of the information of the first fundamental form is required to be fixed.

The stationary untrapped hypersurface boundary conditions [10] provides the "minimal" boundary conditions which are necessary for the presymplectic 
form $\Omega\left(\varphi, \delta \varphi, £_{\xi} \varphi\right)$ to be a total variation for some function $\mathbb{H}(\xi)$, to define energy and angular momentum. Quasilocally, we expect these conserved quantities to depend on the choice of the vector field $\xi$. The stationary untrapped hypersurface fixes the boundary conditions for $\xi$ up to the choice of a quasilocal function of $S$. In the next three sections we shall discuss three such choices which give the Hawking energy, the Brown-York energy and the generalized Hawking energy.

\section{Hawking energy}

For a spherically symmetric stationary untrapped boundary condition, we first pick $\xi$ on $S$ to be [10]

$$
\left.\xi\right|_{S}=h(\varrho) H_{\perp},
$$

where $H_{\perp}$ is the dual expansion vector and $h(\varrho)$ is a quasilocal function of the area element of the (untrapped or marginal) two-surface $S$. Then by equation (44) $£_{\xi \varrho}=0$, the presymplectic form (12) reduced to

$$
\begin{aligned}
0=\Omega\left(\varphi, £_{\xi} \varphi, £_{\xi} \varphi\right) & =-\oint_{S} 2 \varrho h(\varrho)\left(k\left(e_{1}\right) £_{\xi} k\left(e_{1}\right)-k\left(e_{0}\right) £_{\xi} k\left(e_{0}\right)\right) . \\
& =-\oint_{S} \varrho h(\varrho) £_{\xi}\left(k\left(e_{1}\right)^{2}-k\left(e_{0}\right)^{2}\right) . \\
& =£_{\xi} \oint_{S}\left(f(\varrho)-h(\varrho) H^{2}\right) \varrho
\end{aligned}
$$

The Hamiltonian conserved quantity $\mathbb{E}(\xi)$ associated with the vector $\xi$ is given by

$$
\mathbb{E}(\xi)=\oint_{S}\left(f(\varrho)-h(\varrho) H^{2}\right) \varrho .
$$

The free quasilocal functions of the area element, $f(\varrho)$ and $h(\varrho)$, can be chosen such that the expression gives ADM mass at spatial infinity and irreducible mass at marginal surfaces $H=0$. This can be done by letting $f(\varrho)$ to be $1 /(8 \pi \mathbb{R})$ and let $h(\varrho)$ to be $\mathbb{R} /(32 \pi)$, where $\mathbb{R}$ is the area radius given by $\mathbb{R}=\sqrt{\frac{1}{4 \pi} \oint_{S} \varrho}$, then

$$
\mathbb{E}_{H}(\xi)=\frac{\mathbb{R}}{2}\left(1-\frac{1}{16 \pi} \oint_{S} H^{2} \varrho\right),
$$


which is precisely the Hawking energy [20]. In this case the evolution vector $\xi$ on $S$

$$
\left.\xi\right|_{S}=\frac{\mathbb{R}}{32 \pi} H_{\perp}
$$

is precisely the Kodama vector [21].

\section{Brown-York energy}

Alternatively, we can use the unit dual expansion vector. For untrapped surfaces $(|H| \neq 0)$, there is a set of uniquely determined unit normal vectors for the two-surface given by equation (3). If we choose the evolution vector $\xi$ such that $\left.\xi\right|_{S}=\hat{e}_{0}$, this leads to the energy expression[10]

$$
\mathbb{E}(\xi)=\oint_{S}\left(f(\varrho)-k\left(\hat{e}_{1}\right)\right) \varrho
$$

Here we have only one free quasilocal function $f(\varrho)$.

A natural requirement is that the expression should give ADM energy at spatial infinity. By the embedding theorem of Wang and Yau[22, we can embed the two-surface isometrically into Minkowski spacetime, let $k_{0}\left(e_{0}\right)$ and $k_{0}\left(e_{1}\right)$ be the trace of extrinsic curvatures with respect to $e_{0}$ and $e_{1}$, for the two-surface in Minkowski spacetime, then the choice $f(\varrho)=k_{0}\left(\hat{e}_{1}\right)$ gives the Brown-York quasilocal energy [23, 24],

$$
\mathbb{E}_{B Y}=\oint_{S}\left(k_{0}\left(\hat{e}_{1}\right)-k\left(\hat{e}_{1}\right)\right) \varrho .
$$

Note that $\hat{e}_{0}$ fails to be defined in the null case. It seems that this choice is not suitable for the cases involving dynamical black holes.

\section{Generalized Hawking energy}

For an axisymmetric stationary untrapped boundary condition, a natural generalization of the evolution vector field $\xi$ on $S$, is [10]

$$
\left.\xi\right|_{S}=h(\varrho, j) H_{\perp}-\Omega(\varrho, j) \psi,
$$

which is assumed to be timelike or null, where $h(\varrho, j)$ and $\Omega(\varrho, j)$ (angular speed) are functions of the area element $\varrho$ and the angular momentum surface 
density $j$. The angular momentum surface density $j$ is defined by $j=\psi^{A} \varpi_{A}$, where $\psi$ is a vector tangent to $S$ satisfying $\left.i_{\psi} \vartheta^{0}\right|_{S}=0$ and $\left.i_{\psi} \vartheta^{1}\right|_{S}=0$, with the boundary conditions [5, 6, 17, 8, 10, 25],

$$
\left.£_{\psi} \varrho\right|_{S}=0,\left.\quad £_{\psi} H_{\perp}\right|_{S}=-\left.£_{H_{\perp}} \psi\right|_{S}=0 .
$$

The conserved quantity associated with $\psi$ is the angular momentum

$$
\mathbb{J}(\psi)=\frac{1}{8 \pi} \oint_{S} j \varrho=\frac{1}{8 \pi} \oint_{S} \psi^{A} \varpi_{A} \varrho .
$$

The equations (41) and (21) implies $\left.£_{\xi} \varrho\right|_{S}=0$ and $\left.£_{\xi} j\right|_{S}=0$, the "stationary untrapped boundary conditions" [10] are satisfied. The presympectic form (12) then reduced to

$$
0=\Omega\left(\phi, £_{\xi} \phi, £_{\xi} \phi\right)=£_{\xi} \oint_{S}\left(f(\varrho, j)-h(\varrho, j) H^{2}\right) \varrho .
$$

The Hamiltonian conserved quantity $\mathbb{E}(\xi)$ associated with $\xi$ is given by

$$
\left.\mathbb{E}(\xi)=\oint_{S}\left(f(\varrho, j)-h(\varrho, j) H^{2}\right)\right) \varrho .
$$

By requiring the energy expression gives the horizon energy for Kerr black holes at marginal surface $H=0$, we obtain $f(\varrho, j)=\sqrt{\mathbb{R}^{4}+4 \mathbb{J}^{2}} / 8 \pi \mathbb{R}^{3}$. The free quasilocal function $h(\varrho, j)$ can be chosen such that the energy is proportional to the Hawking energy and gives ADM mass at spatial infinity, this implies that $h(\varrho, j)=\sqrt{\mathbb{R}^{4}+4 \rrbracket^{2}} / 32 \pi \mathbb{R}$. Then the Hamiltonian conserved quantity associated with $\xi$,

$$
\mathbb{E}_{G H}=\frac{\sqrt{\mathbb{R}^{4}+4 \mathbb{J}^{2}}}{2 \mathbb{R}}\left(1-\frac{1}{16 \pi} \oint_{S} H^{2} \varrho\right),
$$

is the generalized Hawking energy[10]. The vector on $S$,

$$
\left.\xi\right|_{S}=\frac{\sqrt{\mathbb{R}^{4}+4 \rrbracket^{2}}}{32 \pi \mathbb{R}} H_{\perp}-\Omega \psi
$$

is a generalization of the Kodama vector[10, 21]. 


\section{Acknowledgments}

This work was partially supported by the National Natural Science Foundation of China (10771140), by Shanghai Education Development Foundation (05SG45) and NCTS, Taiwan.

\section{References}

[1] L. B. Szabados, "Quasi-local energy-momentum and angular momentum in GR: a review article", Living Review in Relativity 7 (2004) 4.

[2] S. A. Hayward, Phys. Rev. D 49 (1994) 6467.

[3] A. Ashtekar, C. Beetle, O. Dreyer, S. Fairhurst, B. Krishnan, J. Lewandowski and J. Wisniewski, Phys. Rev. Lett. 85 (2000) 3564.

[4] A. Ashtekar and B. Krishnan, Phys. Rev. Lett. 89 (2002) 261101.

[5] A. Ashtekar and B. Krishnan, Phys. Rev. D 68 (2003) 104030.

[6] E. Gourgoulhon, Phys. Rev. D 72 (2005) 104007.

[7] I. Booth and S. Fairhurst, Class. Quantum Grav. 22 (2005) 4515.

[8] S. A. Hayward, Phys. Rev. D 74 (2006) 104013.

[9] A. Ashtekar and B. Krishnan, "Isolated and dynamical horizons and their applications", Living Review in Relativity 7 (2004) 10.

[10] R. S. Tung, Classical and Quantum Gravity 25 (2008) 085005.

[11] R. S. Tung and H. L. Yu, Physical Review D 78 (2008) 104010.

[12] L. B. Szabados, Class. Quantum Grav. 11 (1994) 1833.

[13] S. C. Anco and R. S. Tung, J. Math. Phys. 43 (2002) 5531; erratum 45 (2004) 2109.

[14] S. C. Anco and R. S. Tung, J. Math. Phys. 43 (2002) 3984; erratum 45 (2004) 2108.

[15] T. Regge and C. Teitelboim, Annals of Physics 88 (1974) 286. 
[16] J. Lee and R. M. Wald, J. Math. Phys. 31 (1990) 725.

[17] J. M. Nester, Mod. Phys. Lett. A 6 (1991) 2655.

[18] R. M. Wald, Phys. Rev. D 48 (1993) 3427.

[19] C. M. Chen, J. M. Nester and R. S. Tung, Phys. Rev. D 72 (2005) 104020.

[20] S. W. Hawking, J. Math. Phys. 9 (1968) 598.

[21] H. Kodama, Prog. Theor. Phys. 63 (1980) 1217.

[22] M.-T. Wang and S.-T. Yau, Phys. Rev. Lett. 102 (2009) 021101.

[23] J. D. Brown, J. W. York, Jr., Phys. Rev. D 47 (1993) 1407.

[24] J. D. Brown, S. R. Lau and J. W. York, Jr., Ann. Phys. (N.Y.) 297 (2002) 175 .

[25] L. B. Szabados, Class. Quantum Grav. 23 (2006) 2291. 